\title{
RNA recognition by a human antibody against brain cytoplasmic 200 RNA
}

\author{
EUIHAN JUNG, ${ }^{1}$ JUNGMIN LEE, ${ }^{1}$ HYO JEONG HONG, ${ }^{2}$ INSOO PARK, ${ }^{3}$ and YOUNGHOON LEE ${ }^{1,4}$ \\ ${ }^{1}$ Department of Chemistry, KAIST, Daejeon 305-701, Korea \\ ${ }^{2}$ Department of Systems Immunology, College of Biomedical Science, Kangwon National University, Chuncheon 200-701, Korea \\ ${ }^{3}$ Molecular Imaging and Therapy Branch, National Cancer Center, Goyang-si 410-769, Korea
}

\begin{abstract}
Diverse functional RNAs participate in a wide range of cellular processes. The RNA structure is critical for function, either on its own or as a complex form with proteins and other ligands. Therefore, analysis of the RNA conformation in cells is essential for understanding their functional mechanisms. However, no appropriate methods have been established as yet. Here, we developed an efficient strategy for panning and affinity maturation of anti-RNA human monoclonal antibodies from a naïve antigen binding fragment (Fab) combinatorial phage library. Brain cytoplasmic 200 (BC200) RNA, which is also highly expressed in some tumors, was used as an RNA antigen. We identified MabBC200-A3 as the optimal binding antibody. Mutagenesis and SELEX experiments showed that the antibody recognized a domain of BC200 in a structure- and sequencedependent manner. Various breast cancer cell lines were further examined for BC200 RNA expression using conventional hybridization and immunoanalysis with MabBC200-A3 to see whether the antibody specifically recognizes BC200 RNA among the total purified RNAs. The amounts of antibody-recognizable BC200 RNA were consistent with hybridization signals among the cell lines. Furthermore, the antibody was able to discriminate BC200 RNA from other RNAs, supporting the utility of this antibody as a specific RNA structure-recognizing probe. Intriguingly, however, when permeabilized cells were subjected to immunoanalysis instead of purified total RNA, the amount of antibody-recognizable RNA was not correlated with the cellular level of BC200 RNA, indicating that BC200 RNA exists as two distinct forms (antibody-recognizable and nonrecognizable) in breast cancer cells and that their distribution depends on the cell type. Our results clearly demonstrate that anti-RNA antibodies provide an effective novel tool for detecting and analyzing RNA conformation.
\end{abstract}

Keywords: RNA recognition; noncoding RNA; BC200 RNA; human monoclonal antibody; breast cancer cells

\section{INTRODUCTION}

Analysis of the human genome led to the surprising revelation that only $2 \%$ of the total genomic sequence comprises protein-coding regions (International Human Genome Sequencing Consortium 2004). Unexpectedly, transcription is prevalent throughout the mammalian genome, yielding complex pools of transcripts, including those with no protein-coding capacity (Carninci et al. 2005). Recent studies have identified several cellular RNAs that function as a class of gene regulators, a role previously assumed to be reserved mainly for proteins (Wilusz et al. 2009; Kugel and Goodrich 2012). In many cases, the biological roles performed by RNAs in cells rely on their three-dimensional structures, although specific sequences have been shown to be essential for function (Chowdhury et al. 2006; Wan et al. 2011, 2012;

\footnotetext{
${ }^{4}$ Corresponding author

E-mail Younghoon.Lee@kaist.ac.kr

Article published online ahead of print. Article and publication date are at http://www.rnajournal.org/cgi/doi/10.1261/rna.040899.113.
}

Breaker 2012). However, effective tools for recognizing the conformations of structured RNA are rare. In general, RNAs are detected via hybridization with complementary nucleic acid probes. However, direct probing of structured RNAs with the classical hybridization method is difficult, since the hybridization procedure requires partial denaturation conditions to secure single-stranded regions for basepairing between RNA and the probe, which could cause conformational changes in RNA. One possible method of effectively probing structured RNAs is the use of specific antibodies. While antibodies against specific proteins can be easily generated, it is considered impossible to produce antibodies recognizing the RNA structure through immunization due to the intrinsic instability of RNA, which leads to rapid degradation upon injection into animals. Furthermore, 
nucleic acids such as RNA or DNA are not normally immunogenic, owing to recognition by immune cells as self-antigens (Pokkuluri et al. 1994), although Stollar (1980) reported that antibodies to single-stranded DNA can be induced by linking them to proteins or polypeptides, followed by injecting to animals. On the other hand, anti-RNA antibodies can be obtained through panning and affinity maturation from an antibody library because previously Piccirilli's group reported the selection of specific antigen binding fragments (Fabs) against a domain derived from the Tetrahymena group I intron using a synthetic phage-display library (Ye et al. 2008; Koldobskaya et al. 2011).

BC200 RNA (brain cytoplasmic 200 RNA) is a small noncoding RNA (Fig. 1) that operates as a translational modulator in human cells (Cao et al. 2006). BC200 RNA is implicated in the inhibition of local synaptodendritic protein synthesis in neurons and is not detected in somatic cells other than neurons (Tiedge et al. 1993). A number of tumors (carcinomas of breast, cervix, esophagus, lung, ovary, parotid, and tongue) are reported to express BC200 RNA (Chen et al. 1997). Moreover, this noncoding RNA appears to be expressed at higher levels in invasive carcinomas than in benign tumors of the breast, suggestive of a role in tumorigenesis (Iacoangeli et al. 2004). However, the biological relevance of high BC200 RNA expression in tumor tissues is yet to be clarified.

In this study, we have developed an efficient strategy for panning and affinity maturation of human monoclonal antibodies binding to RNA from a naïve Fab combinatorial phage library, using BC200 RNA as the antigen. We identified MabBC200-A3 as the optimal binder, which interacted with BC200 RNA at a dissociation constant of $\sim 7 \mathrm{nM}$. Mutagenesis and SELEX experiments showed that the antibody recognizes BC200 RNA in a sequence- and structure-dependent manner. Expression of BC200 RNA in various breast cancer cell lines was further examined using conventional

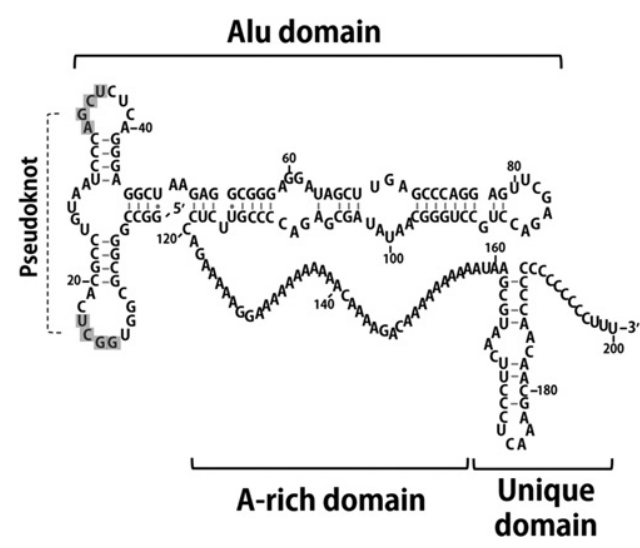

FIGURE 1. Secondary structure model of BC200 RNA. The RNA is composed of a $5^{\prime}$ Alu domain, an internal poly(A) domain, and a $3^{\prime}$ unique domain containing a cytosine-rich stretch. Bases involved in a pseudoknot are shaded. hybridization and immunoanalysis with MabBC200-A3. When total cellular RNAs purified from cells were analyzed, the antibody was able to discriminate BC200 RNA from other RNAs and the amounts of antibody-recognizable BC200 RNA were consistent with hybridization signals among the cell lines. Intriguingly, however, when permeabilized cells were used instead of purified total cellular RNA, the amounts of antibody-recognizable BC200 RNA were different, indicating that BC200 RNA exists as two distinct cellular forms (antibody-recognizable and nonrecognizable) in breast cancer cells. Our results demonstrate the value of the anti-RNA antibody as a novel tool for detecting and analyzing RNA conformation, which cannot be achieved with hybridization.

\section{RESULTS}

\section{Fabs selection against BC200 RNA}

We used a human naïve Fab combinatorial phage library (I Park and HJ Hong, in prep.) to select Fabs recognizing BC200 RNA (Fig. 1). In principle, we adapted the procedures used for the selection of RNA-binding proteins and synthetic antibodies from the groups of Belasco (Laird-Offringa and Belasco 1996) and Piccirilli (Ye et al. 2008; Koldobskaya et al. 2011), respectively. We used a streptavidin-coated immunotube and biotin-tagged BC200 RNA for RNA immobilization, which is known to facilitate more effective RNA binding and clearer separation of buffers than streptavidincoated beads. The specificity of clones against RNA was additionally enhanced by eluting RNA-binding phages from the immunotube with yeast total RNA as competitors. We selected two unique Fab phage clones, FabBC200-A and FabBC200-B, after four rounds of panning (Fig. 2A). Binding of the selected clones to BC200 RNA was confirmed using dot-blot analysis, where an individual phage immobilized on a membrane as a dot was incubated with labeled BC200 RNA. FabBC200-A and FabBC200-B were used to generate soluble whole-antibody forms, MabBC200-A and MabBC200-B, respectively. Determination of their binding affinities with the filter binding assay revealed $K_{\mathrm{d}}$ values of $\sim 36 \mathrm{nM}$ for MabBC200-A and $76 \mathrm{nM}$ for MabBC200-B (Fig. 2B,C). Interestingly, sequence analysis disclosed the same hexapeptide sequence "ARGSPR" on the early CDR3 region of the heavy-chain (CDR-H3) in both clones. Considering that the library originates from human naïve antibodies and rarely has bias toward any specific class of residues, this hexapeptide match appears significant, implying a critical role of the peptide sequence in binding to BC200 RNA.

\section{Affinity maturation of the anti-RNA antibody}

Next, we performed affinity maturation to enhance the affinity of the BC200 RNA-binding antibody, starting with the higher affinity clone, FabBC200-A. For this purpose, a novel 
A

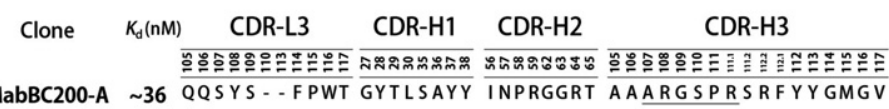
MabBC200-B $\sim 76$ ATWDDSRNGLV GYTLSTYY INSRGGRT ARGSPRLRRDPRRAFDI
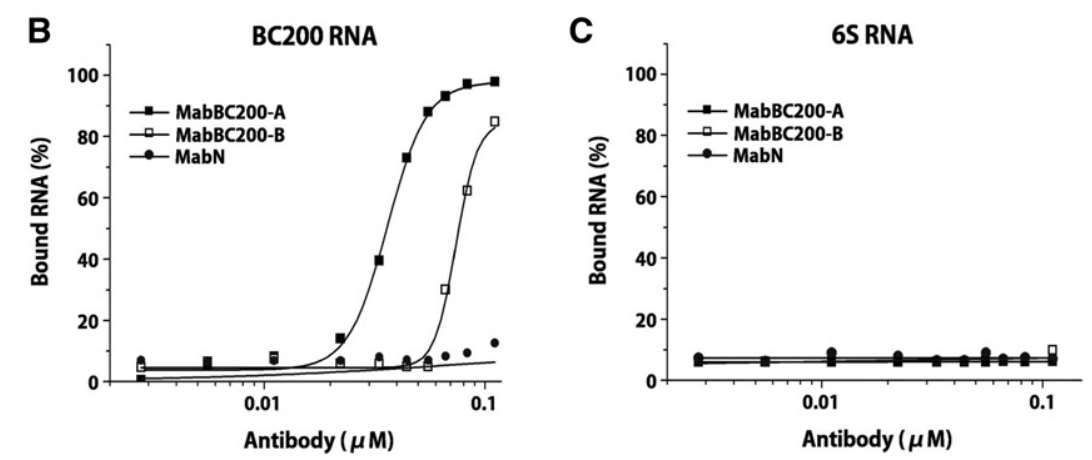

FIGURE 2. Selection of Fabs recognizing BC200 RNA from a human naïve Fab combinatorial phage library. (A) CDR sequences of selected antibodies and dissociation constants $\left(K_{\mathrm{d}}\right)$ of the antibody-RNA complexes. The hexapeptide sequence ARGSPR is underlined. Amino acid numbering and CDR definitions follow the IMGT numbering system (Lefranc 2011). (B) Binding assays for determination of $K_{\mathrm{d}}$ values of the antibody-RNA complexes. Bound RNA signifies RNA retained on the nitrocellulose membrane. Binding reactions were carried out at $25^{\circ} \mathrm{C}$ with $0.6 \mathrm{nM}$ RNA in PBS. (C) 6 S RNA of 184 nt, well-defined, structured RNA derived from Escherichia coli was used as the negative control for the binding assays.

\section{Structural mapping of the RNA- antibody complex}

The highest binder, MabBC200-A3, was selected for further analysis. To identify the regions of $\mathrm{BC} 200$ RNA recognized by the antibody, we performed RNA footprinting using hydroxyl radical (Fig. 4A) and $\mathrm{Pb}^{2+}$ (Supplemental Fig. 2). Notably, two regions of BC200 RNA (residues 7685 and 96-104) were protected by the antibody (Fig. 4B). Within the secondary structure, the two regions were separated by a half-helix turn and were consequently located in the same face for antibody interactions, as shown in a three-dimensional model (Fig. 4C) predicted by Zhao et al. (2012). In addition to these regions, no significant changes in hydroxyl radical or $\mathrm{Pb}^{2+}$ cleavage patterns were observed, suggesting that overall RNA structure remains unchanged upon antibody binding. affinity maturation method was designed (Supplemental Fig. 1). The essential features of this method were sib selection strategy (McCormick 1987) combined with PCR mutagenesis using randomized codons of the nucleotide sequence NNS $(\mathrm{N}=\mathrm{A}, \mathrm{G}, \mathrm{C}$, or $\mathrm{T} ; \mathrm{S}=\mathrm{G}$ or $\mathrm{C})$. The previous affinity maturation study of anti-RNA antibodies screened from a synthetic library disclosed that mutations were observed in the CDR-L3 regions of higher-affinity antibodies at a relatively high frequency, rather than in other CDR regions (Koldobskaya et al. 2011). Accordingly, CDR-L3 of FabBC200-A was chosen as an initial target CDR region for affinity maturation. Nine mutant sets were generated, each containing clones with random mutations in one of nine residues in CDR-L3. The mutant set with the highest affinity against antigen was selected. Individual clones were separated from the selected mutant set and their binding affinities assayed. We simply examined a pool of 96 independent clones from the selected set, which included all 20 amino acids with a probability of $>90 \%$. The mutant set of residue $\mathrm{S} 107$ showed the most improved binding affinity, relative to parental FabBC200-A. Further analysis of 96 clones randomly selected from the S107 mutant pool revealed four clones with higher affinity than the parental clone. Sequence analysis further disclosed S107G, S107A, S107C, and S107V mutations in CDR-L3 (Fig. 3A). Mutant clones were designated FabBC200-A1, FabBC200-A2, FabBC200A3, and FabBC200-A4, respectively. Their whole-antibody forms (MabBC200-As) were generated and tested for binding affinity to BC200 RNA, revealing two- to fivefold enhanced $K_{\mathrm{d}}$ values of 7-18 nM, compared with the parental clone (Fig. 3).

\section{Specificity of the antibody to BC200 RNA}

The structural mapping showed that the region of BC200 RNA protected by the antibody MabBC200-A3 is localized to a structural domain spanning residues $60-110$. To examine how this region is involved in the antibody binding, we generated an RNA fragment of 73 nucleotides (nt), named RNA(51-120), which contained BC200 RNA sequences of residues +51 to +120 and two extra nucleotides at the $5^{\prime}$
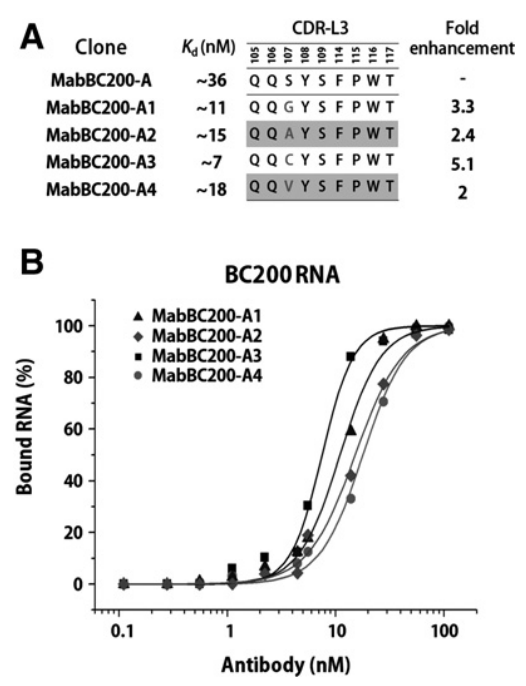

FIGURE 3. Affinity maturation of anti-BC200 RNA antibody. (A) CDR sequences of MabBC200-A-derived and affinity-matured antibodies and $K_{\mathrm{d}}$ of the antibody-RNA complexes. $(B)$ Binding assays for determination of $K_{\mathrm{d}}$ values of complexes between affinity-maturated antibodies and BC200 RNA. Two- to fivefold enhancement was observed. 

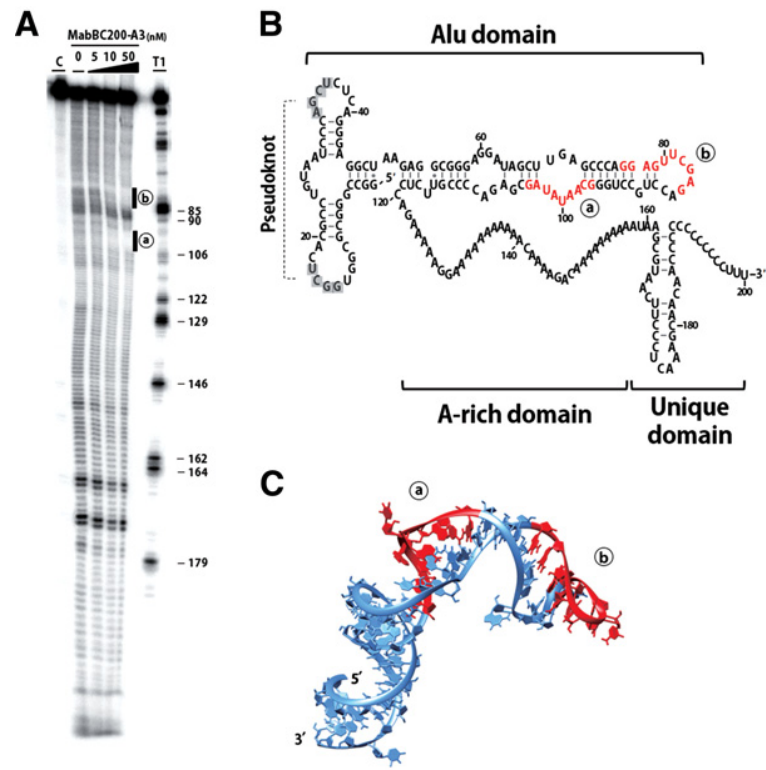

FIGURE 4. Antibody binding regions of BC200 RNA. (A) Hydroxyl radical footprints of BC200 RNA with $0,5,10$, and $50 \mathrm{nM}$ MabBC200-A3. Protected regions are denoted with black bars (a and b). The regions were detected from the range of a $10 \mathrm{nM}$ antibody, consistent with a $K_{\mathrm{d}}$ of $\sim 7 \mathrm{nM}$, as observed using the nitrocellulose filter binding assay. Untreated and RNase T1 ladders of denatured BC200 RNA are shown in lanes $C$ and $T 1$, respectively. $(B)$ Binding regions in the secondary structure of BC200 RNA are highlighted in red letters. (C) Predicted 3D structure of a region of BC200 RNA (residues 51-119) carrying the anti-RNA antibody recognition motif. The binding regions are indicated in red.

end and one extra nucleotide at the $3^{\prime}$ end. The binding assay revealed that RNA(51-120) bound to MabBC200-A3 with a similar affinity to that for BC200 RNA (Fig. 5), suggesting that this truncated version is sufficient for binding to the antibody. To examine which bases in the protected region are important for the RNA-antibody interaction, we performed a SELEX (systematic evolution of ligands by exponential enrichment) (Supplemental Fig. 3; Table 1). First, we chose 11 bases residing on loops in the secondary structure model among the total 19 protected bases. Then we substituted randomized base $\mathrm{N}$ in place of each chosen base for generation of a partially randomized RNA pool derived from RNA(51-120). This RNA pool of $4^{11}\left(4.2 \times 10^{6}\right)$ diversity was subjected to SELEX. RNA species binding to the antibody were selected by immunoprecipitation. After eight rounds of selection, 50 independent clones were isolated and sequenced (Fig. 6; Table 1). Surprisingly, 46 clones had the exactly same sequence of RNA(51-120). The other four clones differed in sequence one another: They had five to 10 mismatches among the 11 bases as compared with RNA(51-120), and they all showed their $K_{d}$ values of $>1 \mathrm{mM}$ for MabBC200-A3. Since their lack of binding was comparable to that of BC200 RNA to the control antibody, we concluded that they had no binding affinity to MabBC200-A3 and that RNA(51-120) is the only one binder out of the partially randomized RNA pool.
Therefore, the SELEX results suggest that all the 11 bases are important for determining specificity of the antibody. Next we tested whether recognition by the antibody would require a specific structure. We performed mutagenesis on RNA(51$120)$ to disrupt a stem formed by base-pairing between residues +71 to +77 and residues +91 to +97 (Fig. 5), which is essential for RNA secondary structure formation. To do this, we substituted 3 nt of +73 to +75 (CCA to GGU) in the middle 3 bp within the stem because the 3 bp does not belong to bases protected by the antibody. While the resulting mutant RNA, RNA(51-120)-M1, did not bind to the antibody, the binding ability of RNA(51-120)-M12 generated by a compensatory mutation in the other strand was restored to the same level as that of RNA(51-120) (Fig. 5), suggesting that the RNA structure is also crucial for the antibody-RNA interaction.

\section{RNA discrimination by the anti-RNA antibody}

Experiments to ascertain whether MabBC200-A3 effectively discriminates BC200 RNA from other RNAs were performed
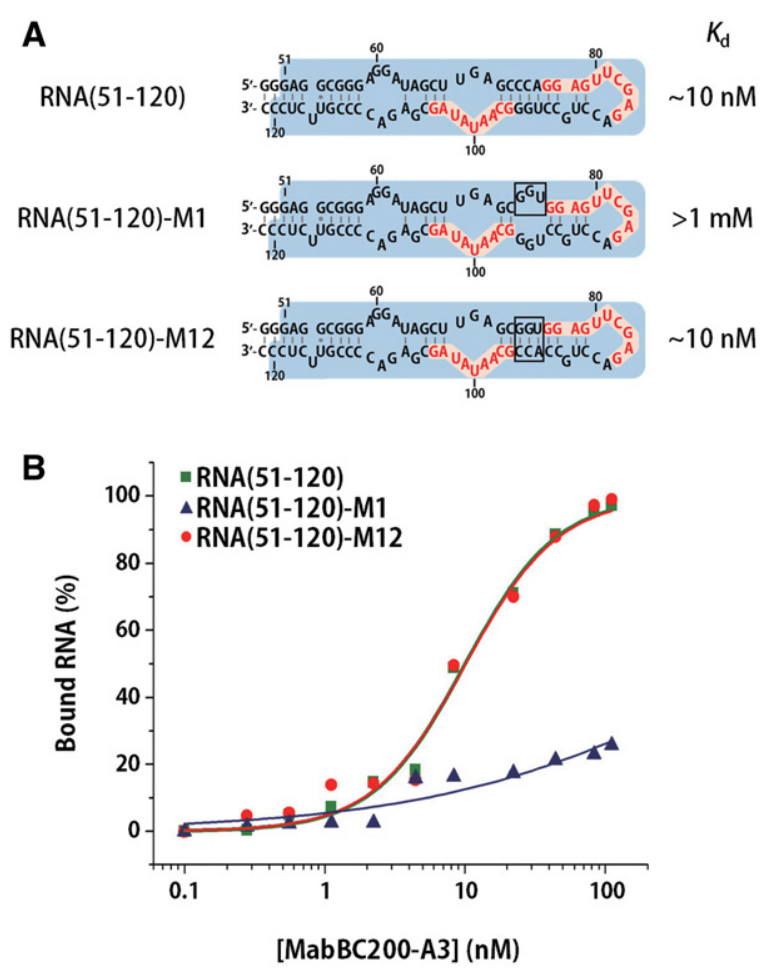

FIGURE 5. Binding affinity of truncated derivatives of BC200 RNA. (A) Possible secondary structures of RNA(51-120) and its derivatives (Zuker 2003), and the $K_{\mathrm{d}}$ of the complexes between RNAs and the MabBC200A3 antibody. RNA(51-120) of 73 nt contains BC200 RNA sequences of residues +51 to +120 , two extra nucleotides at the $5^{\prime}$ end, and one extra nucleotide at the $3^{\prime}$ end. Protected regions by the MabBC200-A3 antibody are highlighted in red letters. The mutated sequences in RNA (51-120)-M1 and RNA(51-120)-M12 are boxed. (B) Binding assays for determination of $K_{\mathrm{d}}$ values of the antibody-RNA complexes were carried out as for Figure 2B. 

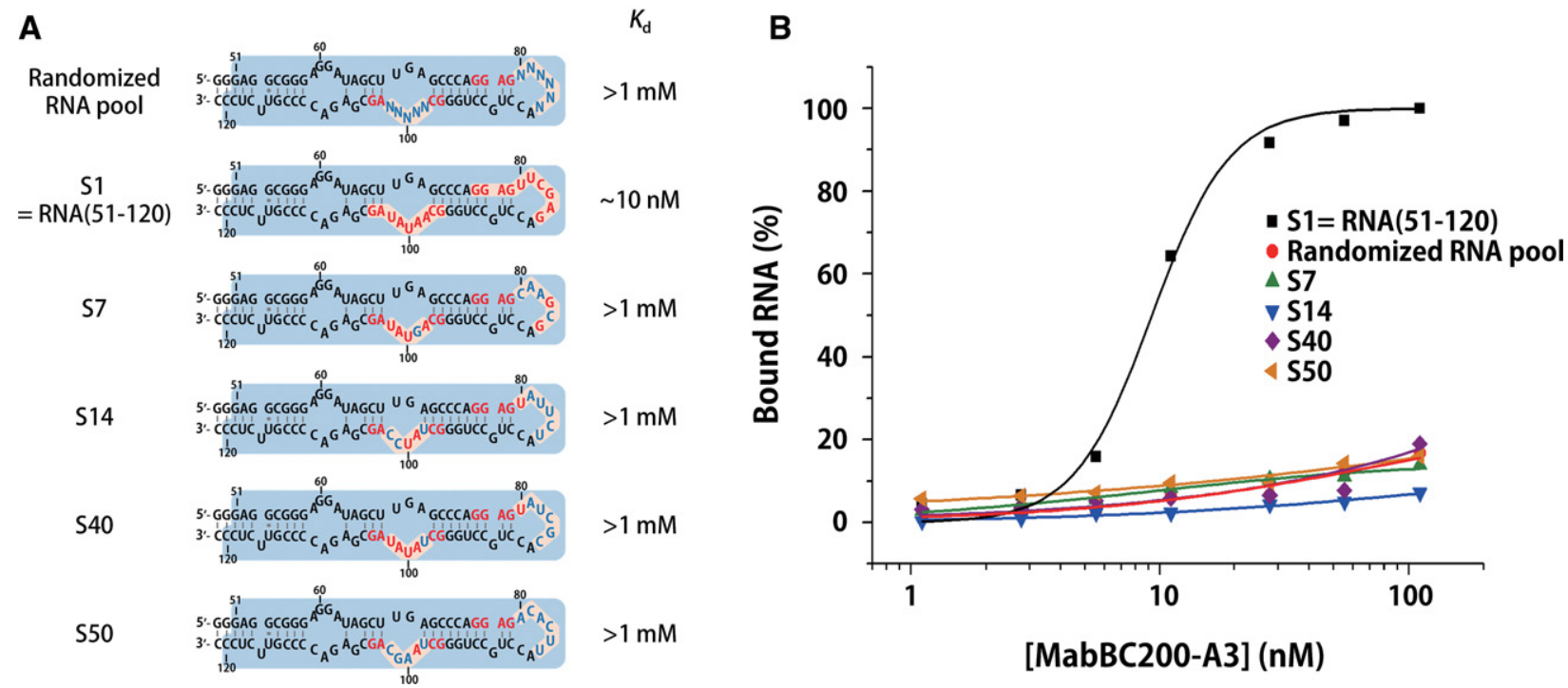

FIGURE 6. Sequence specificity of BC200 RNA for binding to antibody. (A) Possible secondary structures of selected RNAs after eight rounds of selection (Zuker 2003). Regions protected by the MabBC200-A3 antibody are highlighted in red letters. The sequences of the randomized regions are boxed, and the bases that deviated from the BC200 RNA sequences are indicated by blue letters. $(B)$ Binding assays of selected RNAs with the MabBC200-A3 antibody were carried out as for Figure 2B.

using total cellular RNA pools from several breast cancer cell lines. First, BC200 RNA expression in various breast cancer cell lines was examined with Northern blot analysis (Fig. 7A). We observed significant differences in expression levels among the cell lines. BC200 RNA expression increased in the order: MDA-MB-231 $=$ SKBR3 $<$ T47D $<$ Hs578T $<$ MDA-MB-435 < MCF7. MDA-MB-231 and SKBR3 cells showed little expression, similar to the normal breast cell line, MCF10A. Next, the immunoprecipitation assay was performed with total cellular RNAs prepared from these cells (Fig. 7B). Notably, BC200 RNA was immunoprecipitated from BC200 RNA-expressing cells with MabBC200-A3, but not the control antibody. 7SL RNA, a component of the signal recognition particle, is a predecessor of Alu, the most abundant transposable elements in the human genome (Walter and Blobel 1982; Ullu and Tschudi 1984). BC200 RNA is composed of a $5^{\prime}$ Alu domain, which shares high sequence similarity with that of 7SL RNA (Bovia and Strub 1996). While the region of $\mathrm{BC} 200 \mathrm{RNA}$ protected by the MabBC200-A3 antibody was within the Alu domain, 7SL RNA was not precipitated from total RNAs of cells with the antibody, suggesting that MabBC200-A3 specifically recognizes $\mathrm{BC} 200 \mathrm{RNA}$.

Next, to determine whether MabB C200-A3 recognizes BC200 RNA present within the cell, flow cytometric analysis was performed using MabBC200-A3 as primary antibody (Fig. 8A). For this purpose, breast tumor cells were suspended following fixation in formaldehyde and permeabilization with Tween-20 to allow antibody penetration into the cells. The fluorescence intensities determined using flow cytometry were inconsistent

TABLE 1. Sequences of selected RNAs after eight rounds of selection

\begin{tabular}{|c|c|c|}
\hline RNA & Frequency & Sequence $\left(5^{\prime} \rightarrow 3^{\prime}\right)$ \\
\hline RNA(51-120) & - & $\begin{array}{l}\text { GGGAGGCGGGAGGAUAGCUUGAGCCCAGGAGUUCGAGACC } \\
\text { UGCCUGGGCAAUAUAGCGAGACCCCGUUCUCCC }\end{array}$ \\
\hline $\begin{array}{l}\text { Randomized } \\
\text { RNA pool }\end{array}$ & - & $\begin{array}{l}\text { GGGAGGCGGGAGGAUAGCUUGA GCCCAGGAGNNNNNNACC } \\
\text { UGCCUGGGCNNNNNAGCGAGACCCCGUUCUCCC }\end{array}$ \\
\hline S1 & 46 & $\begin{array}{l}\text { GGGAGGCGGGAGGAUAGCUUGA GCCCAGGAGUUCGAGACC } \\
\text { UGCCUGGGCAAUAUAGCGA GACCCCGUUCUCCC }\end{array}$ \\
\hline S7 & 1 & $\begin{array}{l}\text { GGGAGGCGGGAGGAUAGCUUGA GCCCAGGAGCAAGCGACC } \\
\text { UGCCUGGGCAGUAUAGCGA GACCCCGUUCUCCC }\end{array}$ \\
\hline S14 & 1 & $\begin{array}{l}\text { GGGAGGCGGGAGGAUAGCUUGA GCCCAGGAGUAUUCUACC } \\
\text { UGCCUGGGCUAUCCAGCGA GACCCCGUUCUCCC }\end{array}$ \\
\hline S40 & 1 & $\begin{array}{l}\text { GGGAGGCGGGAGGAUAGCUUGA GCCCAGGAGUAUCGCACC } \\
\text { UGCCUGGGCUAUAUAGCGA GA CCCCGUUCUCCC }\end{array}$ \\
\hline S50 & 1 & $\begin{array}{l}\text { GGGAGGCGGGAGGAUAGCUUGAGCCCAGGAGACACUUACC } \\
\text { UGCCUGGGCUAAGCAGCGAGACCCCGUUCUCCC }\end{array}$ \\
\hline
\end{tabular}

After eight rounds of SELEX, selected RNAs were subcloned and sequenced. Protected regions by the MabBC200-A3 antibody are highlighted in bold. The sequences of the randomized regions are boxed, and the bases that deviated from the BC200 RNA sequences are indicated by shaded letters. 

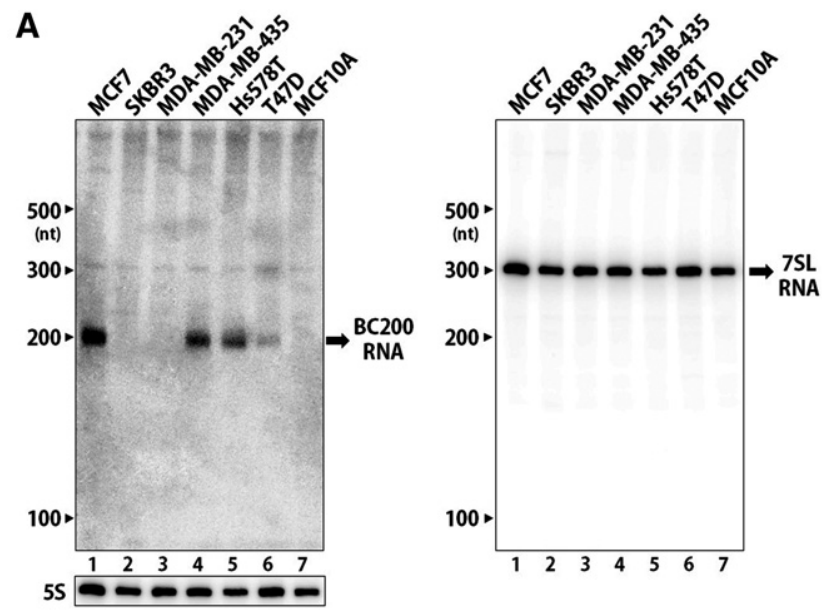

B

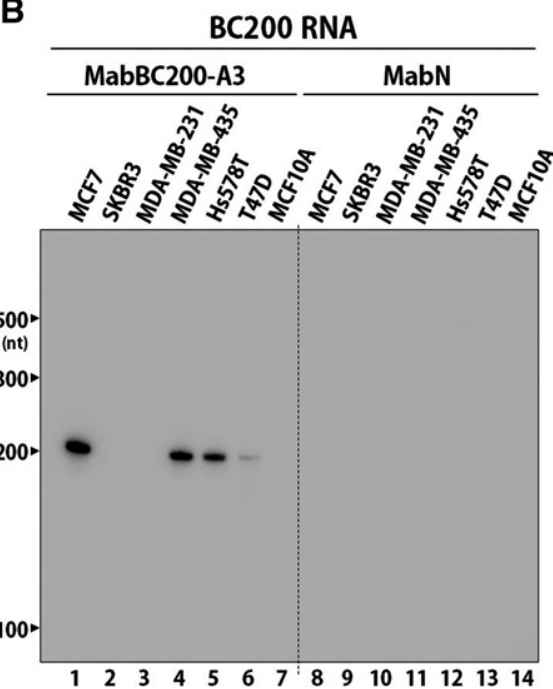

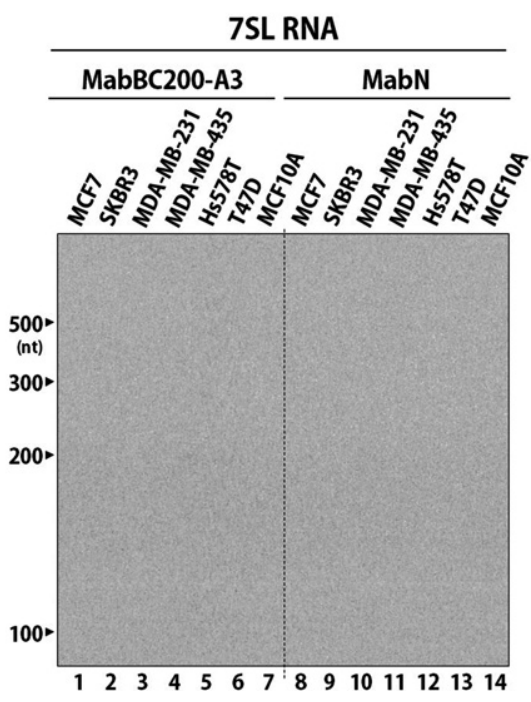

FIGURE 7. RNA recognition by MabBC200-A3. (A) Total cellular RNAs purified from breast cancer cell lines (MCF7, SKBR3, MDA-MB-231, MDA-MB-435, Hs578T, and T47D) and a normal breast cell line, MCF10A, were analyzed for BC200 RNA content via Northern blot. The membranes were hybridized with the BC200 RNA probe (left) or 7SL RNA probe (right). (B) Total cellular RNAs were immunoprecipitated with MabBC200-A3. The BC200 RNA (left) or 7SL RNA (right) content in immunoprecipitates was analyzed using Northern blot, with $\mathrm{MabN}$ as a negative control antibody.

with Northern signals of BC200 RNA in the cells (Fig. 8B). Northern signals representing the cellular content of BC200 RNA increased in the order: T47D $<$ Hs578T $<$ MDA-MB $-435<$ MCF7, while the order of fluorescence signals was MDA-MB-435 $<$ T47D $<$ MCF7 $<$ Hs578T. To confirm that the flow cytometry signals result from BC200 RNA-antibody complexes, the complexes were immunoprecipitated directly from cell lysates and the BC200 RNA content analyzed using Northern blotting (Fig. 9A) and RT-PCR (Supplemental Fig. S4). Comparative data revealed that BC200 RNA levels immunoprecipitating from different breast cancer cells are consistent with flow cytometry signals (Fig. 9B). These results suggest that BC200 RNA exists as two forms in the cell: one that is recognized by MabBC200-A3 and another that is not recognized by the antibody. Interestingly, while the two estrogen receptor (ER)-negative cell lines, MDA-MB-435 and Hs578T (Neve et al. 2006), expressed comparable amounts of BC200 RNA, the amounts of BC200 RNA recognized by MabBC200-A3 were distinct between the two cell lines. The majority of antigen was not recognized by MabBC200-A3 in MDA-MB-435, while Hs578T cells contained the highest amount of antibody-binding BC200 RNA.

\section{DISCUSSION}

In the present study, we developed an efficient strategy for panning and affinity maturation of human monoclonal antibodies binding to RNA from a naïve Fab combinatorial phage library. Especially, sib selection combined with PCR mutagenesis using randomized NNS codons was successfully utilized for affinity maturation. We expect that our affinity maturation method should be faster than the existing protocols using libraries that are newly constructed through mutagenesis or chain shuffling across antibodies (Gram et al. 1992; Schier et al. 1996). While the diversity of the clones examined with our maturation method is relatively low compared with that obtained with the new library construction methods using mutagenesis or shuffling, the selected set has a greater probability of containing clones having higher affinity.

We identified antibodies that recognize structural motifs of BC200 RNA, implying that naïve $\mathrm{B}$ cells have clones capable of RNA recognition. Footprinting experiments performed with the best binder, MabBC200-A3, revealed that the antibody exclusively binds to a specific region of BC200 RNA. The SELEX and mutagenesis data further demonstrated that MabBC200-A3 specifically recognizes BC200 RNA by binding this domain in a sequence- and structure-dependent manner. When MabBC200-A3 was tested with total RNAs purified from various breast cancer cell lines, immunoanalytical signals for BC200 RNA coincided with conventional hybridization signals. Furthermore, the antibody was able to discriminate BC200 RNA from other RNAs in the purified total RNA, supporting its usefulness as a specific RNA structure-recognizing probe for the RNA. Intriguingly, however, when permeabilized cells were used instead of purified total cellular RNA, 


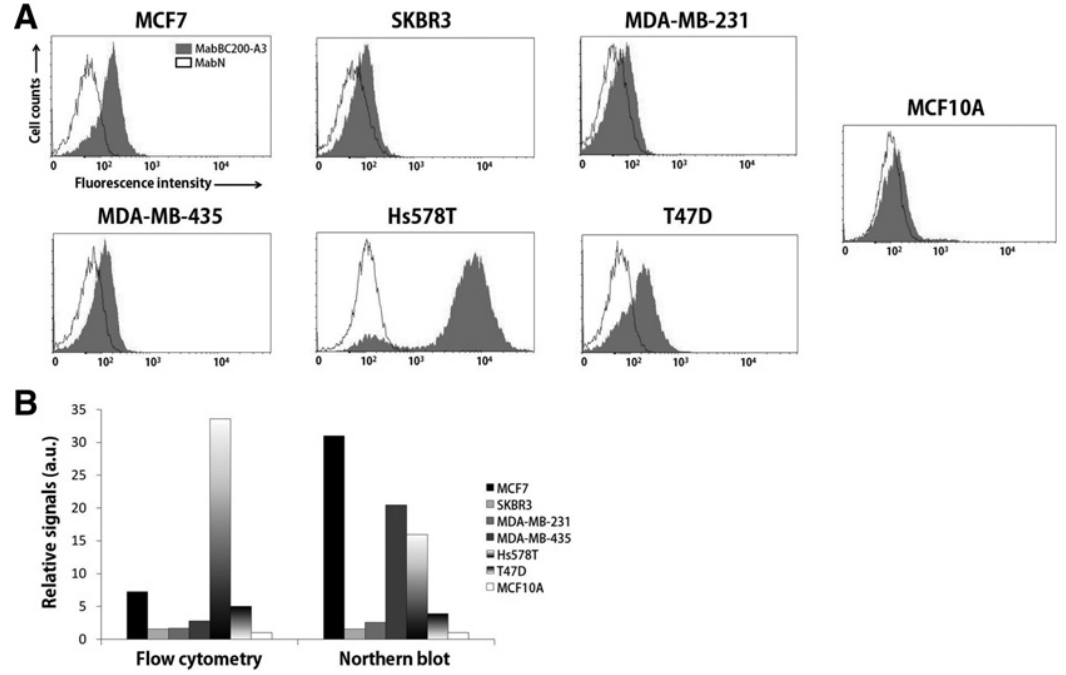

FIGURE 8. Flow cytometric analysis. (A) Fixed and permeabilized cells were treated with MabBC200-A3, followed by FITC-conjugated anti-human IgG, and subjected to flow cytometric analysis. Plots for cells treated with MabBC200-A3 and MabN are indicated by shaded and white areas, respectively. (B) Comparison between the flow cytometric signals of $A$ and the Northern blot data of Figure 7A. Levels were normalized with MabN and 5S for flow cytometric and Northern blot analyses, respectively. The relative signals are presented in arbitrary units for each cell.

the amounts of antibody-recognizable BC200 RNA were different, indicating that BC200 RNA exists as two distinct forms (antibody-recognizable and nonrecognizable) in the cell and that their distribution depends on the cell type. For BC200 RNA recognition by the antibody, its structure should maintain the integrity of the antigenic motif, and antibody binding to this motif should not be hindered by other BC200 RNA-binding proteins (Fig. 10). The functional differences between BC200 RNA recognized by the antibody and that lacking antibody-binding affinity remain to be established.

RNA function depends on both its conformation and sequence. Furthermore, RNA is a molecule displaying complexity and dynamic behavior that exists in various conformations and as different ribonucleoproteins in cells. Therefore, detecting the presence or absence of free RNA motifs in cells is essential for understanding the cellular functions of RNA. In this respect, RNA structure-recognizing antibodies can potentially overcome the limitations of complementarity-based RNA detection through hybridization, which prevents retention of structure. In addition, all existing methods of antibody technology can be applied for enhancing utilization of RNA structure-recognizing antibodies in basic and applied research. For instance, given that RNA usually folds into extensive structures, an anti-RNA antibody may provide an innovative tool for dissecting RNA motifs with specific conformations. The specific motifs recognized by the anti-RNA antibody may act as potent biomarkers for diagnosis. Moreover, antibody delivery systems into living cells are currently under development (Kuo et al. 1998; Fang et al. 2007), and cells expressing disease-related RNAs may thus be targeted by anti-RNA antibodies more effectively than antisense nucleic acids.

\section{MATERIALS AND METHODS}

\section{In vitro preparation of RNA}

A DNA template for BC200 RNA was obtained from human genomic DNA (Roche) via PCR using a primer pair of BC200-T7up (5'-GAATTCTAATACGACTCACTATA GGCCGGGCGCGGTG)/BC200-dn (5'-CCC AAGCTTTTTAAAGGGGGGGGGGGGTTG TTG) and cloned into the EcoRI and HindIII sites of pUC19 (New England Biolabs). The resulting plasmid DNA was cleaved with DraI and used as a template for in vitro transcription with T7 RNA polymerase (Promega) to generate in vitro transcripts having the same $5^{\prime}$ and $3^{\prime}$ ends as the natural BC200 RNA. Truncated BC200 RNA derivatives, RNA(51-120), RNA(51-120)-M1, and RNA(51-120)-M12 were generated similarly. Briefly, the DNA template for RNA(51-120) was amplified via PCR using a primer pair, RNA(51-120)-T7-up (5'-CCGGAATTCTAATACGACTCACTATA GGGAGGCGGGAGGATAGC)/RNA(51-120)-dn (5'-TCCCCCGG
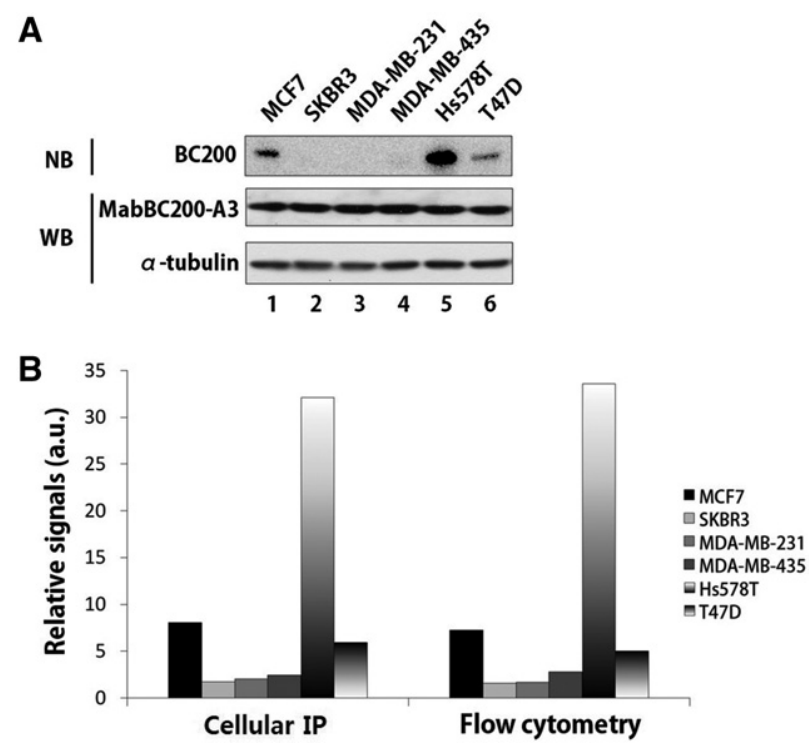

FIGURE 9. Immunoprecipitation of BC200 RNA. (A) Lysates from cells treated with MabBC200-A3 were subjected to immunoprecipitation. The BC200 RNA content in precipitates was analyzed via Northern blot using the BC200 RNA probe. It was confirmed via Western blot with lysates of antibody-treated cells that all the cell lines were permeable to antibodies to similar extents. After electrophoresis on a 5\% SDS-PAGE gel, the lysates were blotted with MabBC200-A3 or anti-tubulin antibody ( $\alpha$-tubulin). NB and WB indicate Northern blot and Western blot, respectively. (B) Comparison between the NB data after cellular immunoprecipitation (Cellular IP) of $A$ and the flow cytometric signals of Figure 8A. 

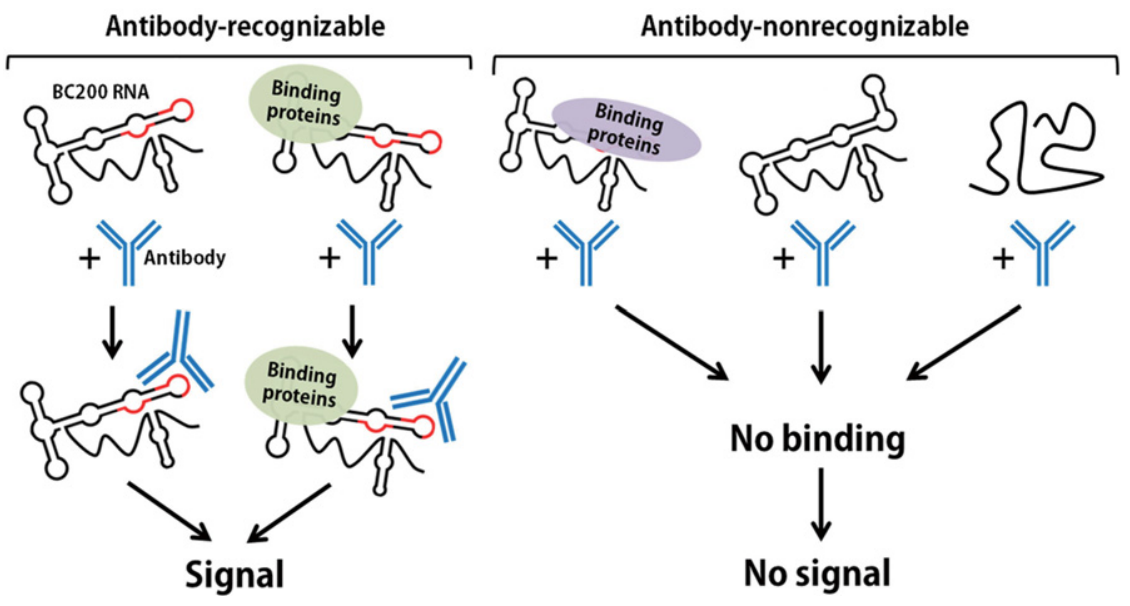

FIGURE 10. A human anti-RNA antibody (blue) binding to a specific structural motif (red) of BC200 RNA. BC200 RNA exists as two distinct cellular forms (antibody-recognizable and nonrecognizable), depending on the structural integrity of the antigenic RNA motif and its accessibility to the antibody.

GAGAACGGGGTCTCGC). The DNA template for RNA(51-120)M1 was amplified using a primer pair, RNA(51-120)-M1-up (5'-GG ATAGCTTGAGCGGTGGAGTTCGAGACCTGCC)/RNA(51-120)M1-dn (5'-GGCAGGTCTCGAACTCCACCGCTCAAGCTATCC). The DNA template for RNA(51-120)-M12 was amplified via recombinant PCR using two primer pairs, RNA(51-120)-T7-up/RNA(51120)-M1-dn and RNA(51-120)-M12-PCR2-up (5'-GGAGTTCGAG ACCTGCCACCGCAATATAGCGAGACC)/RNA(51-120)-dn. The amplified DNA fragments were cloned into the EcoRI and SmaI sites of pUC19. The resulting plasmid DNAs were cleaved with SmaI and used as templates for in vitro transcription with T7 RNA polymerase.

The DNA template for an RNA(51-120)-derived library for SELEX was obtained via recombinant PCR using two primer pairs, RNA(51-120)-T7-up/RNA(51-120)-SELEX-dn (5'-GCCCAGGCA GGTNNNNNNCTCCTGGGCTCAAGCTATCCTCCCGCCTCCC TATAGTGAGTCGTATTAGAATTCCGG) and RNA(51-120)SELEX-up (5'-GATAGCTTGAGCCCAGGAGNNNNNNACCTGCC TGGGCNNNNNAGCGAGACCCCGTTCTCCCGGGGGA)/RNA (51-120)-dn and amplified via PCR using a primer pair, RNA (51-120)-T7-up/RNA(51-120)-dn. The amplified DNA was cleaved with SmaI and subjected to in vitro transcription with T7 RNA polymerase to generate the RNA(51-120)-derived library. After selection, cDNAs were generated via RT-PCR using a primer pair, RNA(51-120)-T7-up/RNA(51-120)-dn, and were again cleaved with SmaI and subjected to in vitro transcription for additional rounds of selection.

All the in vitro transcripts were purified via gel elution. Before use RNA was renatured by heating for $5 \mathrm{~min}$ at $65^{\circ} \mathrm{C}$, chilling on ice for $10 \mathrm{~min}$, and slowly returning to room temperature.

To prepare a biotinylated BC200 RNA for panning experiments, a DNA fragment was amplified by PCR with a primer pair of BC200T7-up/BC200B-dn (5'-CTTCCGGATCCGACCGTGGTGCCCTT GCGGGCAGAAGTCCAAATGCGATCCAAAGGGGGGGGGGGG) and cloned into pUC19 EcoRI and BamHI. The resulting plasmid DNA was cleaved with $S t u$ I and used as the DNA template for in vitro transcription. The in vitro transcript and a $5^{\prime}$ biotinlyated oligonucleotide (5'-AGGATCCGACCGTGGTGCCC) were mixed in the ratio of $1: 2.5$ and incubated for $5 \mathrm{~min}$ at $85^{\circ} \mathrm{C}$, chilled on ice for
$10 \mathrm{~min}$, and slowly returned to room temperature to anneal through the extra adaptor sequence of RNA at the $3^{\prime}$ end as well as for RNA renaturation.

\section{Selection of human Fab}

For selection of Fab phage clones via panning, the phagemid vector, Fab1, was used to construct phage display libraries with a repertoire of human $\mathrm{V}_{\mathrm{L}}$ or $\mathrm{V}_{\mathrm{H}}$. The resulting Fab libraries had $5.0 \times 10^{8}$ diversity. The libraries were panned for four rounds for biotinylated BC200 RNA, which was immobilized on a streptavidin-coated immunotube at room temperature. Briefly, phages were preincubated three times with the immunotube and 100 pmol 5' biotinylated DNA oligonucleotide for $30 \mathrm{~min}$ to remove false-positive binders, such as streptavidin-, biotin-, or the oligonucleotide-binders, followed by incubation with 10 pmol BC200 RNA-immobilized immunotubes for $30 \mathrm{~min}$. The immunotube was washed 10 times with $\mathrm{PBS}(8 \mathrm{mM}$ $\mathrm{Na}_{2} \mathrm{HPO}_{4}, 1.5 \mathrm{mM} \mathrm{KH}_{2} \mathrm{PO}_{4}, 137 \mathrm{mM} \mathrm{NaCl}$, and $3 \mathrm{mM} \mathrm{KCl}$ ) containing $0.05 \%$ Tween- 20 and eluted with $5 \mathrm{mg}$ yeast total RNA to recover RNA-binding Fabs. Washing was increased 10 times every panning round. After each round of selection, recovered phages were amplified with VCS M13 helper phage at 20-fold MOI. Phages were purified through PEG precipitation, and the affinity for BC200 RNA was measured with dot-blot assays. Briefly, each clone phage was transferred to nitrocellulose membrane using Minifold dot-blot apparatus (Whatman) with ${ }^{32} \mathrm{P}$-labeled BC200 RNA as a probe. The membrane was exposed to imaging plate BAS-IP (Fuji) and analyzed on a phosphor-image analyzer, FLA7000 (Fuji). Positive clones were sequenced.

\section{Expression and purification of whole antibody forms}

$\mathrm{V}_{\mathrm{H}}$ and $\mathrm{V}_{\mathrm{L}}$ of selected Fabs were fused with Ig heavy- and light-chain leader sequences (Ryu et al. 1996) using PCR, respectively. Amplified products were digested with EcoRI and ApaI for heavy-chain and HindIII and BstXI for light-chain and subcloned into the corresponding sites of pdCMV-dhfrC-A10A3 (Lee et al. 2012). The resulting expression plasmid was transfected into human embryonic kidney (HEK) 293T cells using Lipofectamine 2000 (Invitrogen), according to the manufacturer's instructions. Transfected cells were cultured in protein-free CD293 medium (Invitrogen), and culture supernatants were subjected to affinity chromatography on a Protein G-Agarose (Invitrogen) column. The purity of the antibody was determined using SDS-PAGE, and concentrations were measured using protein assay dye reagent (Bio-Rad), according to the manufacturer's instructions.

\section{Filter binding assays}

RNA was renatured by heating for $5 \mathrm{~min}$ at $65^{\circ} \mathrm{C}$, chilling on ice for $10 \mathrm{~min}$, and slowly returning to room temperature. RNA-antibody complexes were formed in $100 \mu \mathrm{L}$ PBS containing $0.6 \mathrm{nM}$ RNA, 
$2 \mathrm{nM}-100 \mathrm{nM}$ antibodies, and 2 units/ $\mu \mathrm{L}$ RNase inhibitor (Enzynomics) for $30 \mathrm{~min}$ at room temperature. The reaction mixture was applied to a dot-blot apparatus in which Hybond ECL membrane (GE Healthcare) and Hybond-XL membrane (GE Healthcare) were layered with Whatman $3 \mathrm{MM}$ paper in between. In this process, RNAs that passed through the Hybond ECL membrane without binding to the antibody were immobilized on the Hybond-XL membrane. The Hybond-XL membrane was washed two times with PBS and hybridized with $5^{\prime}-{ }^{32} \mathrm{P}-$ labeled antisense oligonucleotide probe $\left(5^{\prime}\right.$-TTTGAGGGAAGTTACGCTTAT for BC200 RNA, 5'-GGGAGAACGGGGTCTCGC for truncated BC200 RNA derivatives, or 5'-GGGAATCTCCGAGATGCCGCC for Escherichia coli 6S RNA as an irrelevant RNA), exposed to the imaging plate, and analyzed on the Phosphor-image analyzer. The amount of radiolabeled RNA on each filter was quantified with ImageJ software (NIH), and plots of bound fractions of RNA versus concentration of antibody were obtained with Origin software (OriginLab). Binding constants $\left(K_{\mathrm{d}}\right)$ were calculated by fitting data to the Hill equation.

\section{Affinity maturation}

To enhance affinity for RNA, we implemented sib selection combined with PCR mutagenesis using randomized codons. Each of the nine residues of CDR-L3 of FabBC200-A was randomly mutated via PCR with primers containing the NNS codon. PCR products were subcloned into the BstXI site of Fabl. After the transformation of $E$. coli TG1 cells, each mutant set was transformed into phages. The resulting mutant phage sets were analyzed for affinity using dot-blot assays. A set with mutation at the third residue of CDRL3 showed the highest affinity for RNA. Cells of the selected mutant set were grown in $2 \times$ YTA plates, and 96 individual clones were randomly obtained. The following equation shows that the 96 clones cover all 20 amino acids with a probability of $>90 \%$ :

$$
\begin{aligned}
P= & \frac{1}{X^{N}} \times\left(X^{N}-\left(x C x-1 \times(x-1)^{N}-x C x-2 \times(x-2)^{N}\right.\right. \\
& +-+-\ldots))
\end{aligned}
$$

where $P$ is probability for a pool containing all independent clones, $X$ represents independent clones to be covered, and $N$ is the number of clones to be tested. Four clones displaying higher affinity than the original clone were subjected to sequence analysis as well as filter binding assays to determine dissociation constants $\left(K_{\mathrm{d}}\right)$.

\section{RNA footprinting}

Hydroxyl radical footprinting analysis was conducted using the Fe/ EDTA-mediated Fenton reaction. Experiments were performed as described previously (Koldobskaya et al. 2011). Briefly, BC200 RNAs were $3^{\prime}$ end-labeled with $\left[{ }^{32} \mathrm{P}\right] \mathrm{pCp}$ using T4 RNA ligase (New England Biolabs). Labeled RNA molecules were renatured by heating for $5 \mathrm{~min}$ at $65^{\circ} \mathrm{C}$, chilling on ice for $10 \mathrm{~min}$, and slowly returning to room temperature. Next, $1 \mu \mathrm{L}$ of $5 \mathrm{mg} / \mathrm{mL}$ heparin, $1 \mu \mathrm{g}$ yeast tRNA, and the corresponding antibody were added to labeled RNA, yielding a total reaction volume of $7 \mu \mathrm{L}$. This binding reaction was carried out in $1 \times$ PBS for $30 \mathrm{~min}$ at room temperature. Subsequently, $1 \mu \mathrm{L}$ of $1 \mathrm{mM} \mathrm{Fe}(\mathrm{II})\left(\mathrm{NH}_{4}\right)_{2}\left(\mathrm{SO}_{4}\right)_{2}, 1 \mu \mathrm{L}$ of 1.25 $\mathrm{mM}$ EDTA, and $1 \mu \mathrm{L}$ of $60 \mathrm{mM}$ sodium ascorbate were added, and the reactions were incubated for $30 \mathrm{~min}$ at room temperature.
Reactions were terminated by adding $1 \mu \mathrm{L}$ of $100 \mathrm{mM}$ thiourea and $11 \mu \mathrm{L}$ gel loading buffer II (Ambion). Samples were denatured for $5 \mathrm{~min}$ at $95^{\circ} \mathrm{C}$ and loaded on a $5 \%(\mathrm{v} / \mathrm{v})$ polyacrylamide-9 M urea sequencing gel. RNase T1 ladders were obtained according to the manufacturer's instructions (Ambion).

\section{SELEX}

The RNA(51-120)-derived library of $4^{11}\left(4.2 \times 10^{6}\right)$ diversity was used for SELEX. In the first round of selection, RNA (0.2 ng, $\sim 5 \times 10^{9}$ molecules) in $50 \mu \mathrm{L}$ PBS was preincubated with $10 \mu \mathrm{L}$ Protein G-Agarose for $30 \mathrm{~min}$ to remove false-positive binders, followed by mixing with $2.8 \mathrm{nM}$ MabBC200-A3 in $100 \mu \mathrm{L}$ PBS and incubated for $30 \mathrm{~min}$ at room temperature. The RNA-antibody complex was immunoprecipitated with Protein G-Agarose. The immunoprecipitates were washed five times with PBS containing $0.05 \%$ Tween-20 and were phenol-extracted. The RNA was recovered from ethanol precipitation and amplified through RT-PCR. The amplified cDNA was used to generate the RNA pool for additional rounds of selection. After the eighth round of selection, the amplified cDNA was cloned into T-blunt vector (SolGent). Individual cDNA clones were identified by DNA sequencing.

\section{Northern blot analysis}

Total cellular RNA was extracted from cells with the easy-blue kit (Intron), according to the manufacturer's instructions. For Northern blot analysis, RNA samples were electrophoresed on a $6 \%$ polyacrylamide gel containing $7 \mathrm{M}$ urea and electrotransferred to Hybond-XL membrane (GE Healthcare). Hybridization was carried out with the $5^{\prime}-{ }^{32} \mathrm{P}-$ labeled BC200 RNA antisense oligonucleotide probe, as previously described (Lee et al. 2013). 7SL RNA and 5S RNA were probed with $5^{\prime}-{ }^{32} \mathrm{P}$-labeled 7SL $\left(5^{\prime}\right.$-GAGGTCACCAT ATTGATGCCGAACTTAGTG) and 5S (5'-CATCCAAGTACTAA CCAGGCCC) antisense oligonucleotide. The membrane was analyzed as described in the filter binding section.

\section{Immunoprecipitation}

After washing in ice-cold PBS, collected cells were resuspended in PBS containing 10\% fetal bovine serum (Invitrogen). Cells were fixed in $0.1 \%$ formaldehyde for $15 \mathrm{~min}$ at room temperature, permeabilized in PBS containing 0.1\% Tween-20, and subsequently incubated with MabBC200-A3 for 30 min at room temperature. After every step, cells were washed twice with ice-cold PBS. Collected cells were resuspended in ice-cold RIPA lysis buffer $(150 \mathrm{mM} \mathrm{NaCl}, 1 \%$ sodium deoxycholate, $0.1 \%$ SDS, $1 \%$ Triton X-100, 2 mM EDTA, and $50 \mathrm{mM}$ Tris- $\mathrm{HCl}$ at $\mathrm{pH} 7.5$ ), incubated on ice for $30 \mathrm{~min}$, and recentrifuged for $20 \mathrm{~min}$ at 16,000g. The supernatant fractions were used as cell lysates for immunoprecipitation with Protein GAgarose. Immunoprecipitates were analyzed via Northern blot for BC200 RNA and 7SL RNA content. Whole-cell lysates used as input were prepared using the same procedure, except for antibody treatment. To measure the levels of input antibodies, cell lysates were prepared without the final washing procedure and subjected to Western blot analysis. Where necessary, purified total cellular RNAs were subjected to immunoprecipitation. Briefly, purified total RNA was renatured, as described with in vitro transcripts above, and 
incubated with MabBC200-A3 in $50 \mu \mathrm{L}$ PBS for $30 \mathrm{~min}$ and immunoprecipitated with Protein G-Agarose. The immunoprecipitates were phenol-extracted, and the BC200 RNA content in the precipitates was analyzed using Northern blot.

\section{Flow cytometry}

Procedures for cell preparations and primary antibody treatments were the same as those for immunoprecipitation. Cells were subsequently treated with fluorescein isothiocyanate (FITC)-conjugated anti-human IgG (Bethyl) for $30 \mathrm{~min}$ at room temperature in the dark. Collected cells were filtered with Strainer capped tubes (BD) and analyzed using the LSRII flow cytometry system (BD). Plots were obtained using Flowing software (Cell Imaging Core).

\section{SUPPLEMENTAL MATERIAL}

Supplemental material is available for this article.

\section{ACKNOWLEDGMENTS}

We thank Dr. Dae-Hee Kim for helpful discussions. This work was initiated by the KAIST High-Risk High-Return Project (HRHRP) and subsequently supported by a National Research Foundation of Korea (NRF) Grant from the Korean government (MSIP; 20100029167, 2011-0020322), and also by the Intelligent Synthetic Biology Center of the Global Frontier Project (funded by MSIP; 2012M3A6A8055678).

Received June 25, 2013; accepted February 19, 2014.

\section{REFERENCES}

Bovia F, Strub K. 1996. The signal recognition particle and related small cytoplasmic ribonucleoprotein particles. J Cell Sci 109: 2601-2608.

Breaker RR. 2012. Riboswitches and the RNA world. Cold Spring Harb Perspect Biol 4: a003566.

Cao X, Yeo G, Muotri AR, Kuwabara T, Gage FH. 2006. Noncoding RNAs in the mammalian central nervous system. Annu Rev Neurosci 29: 77-103.

Carninci P, Kasukawa T, Katayama S, Gough J, Frith MC, Maeda N, Oyama R, Ravasi T, Lenhard B, Wells C, et al. 2005. The transcriptional landscape of the mammalian genome. Science 309: 15591563.

Chen W, Bocker W, Brosius J, Tiedge H. 1997. Expression of neural BC200 RNA in human tumours. J Pathol 183: 345-351.

Chowdhury S, Maris C, Allain FH, Narberhaus F. 2006. Molecular basis for temperature sensing by an RNA thermometer. EMBO $J$ 25: $2487-2497$.

Fang J, Yi S, Simmons A, Tu GH, Nguyen M, Harding TC, VanRoey M, Jooss K. 2007. An antibody delivery system for regulated expression of therapeutic levels of monoclonal antibodies in vivo. Mol Ther 15: $1153-1159$.

Gram H, Marconi LA, Barbas CF III, Collet TA, Lerner RA, Kang AS. 1992. In vitro selection and affinity maturation of antibodies from a naive combinatorial immunoglobulin library. Proc Natl Acad Sci 89: 3576-3580.

Iacoangeli A, Lin Y, Morley EJ, Muslimov IA, Bianchi R, Reilly J, Weedon J, Diallo R, Bocker W, Tiedge H. 2004. BC200 RNA in invasive and preinvasive breast cancer. Carcinogenesis 25: 2125-2133.
International Human Genome Sequencing Consortium. 2004. Finishing the euchromatic sequence of the human genome. Nature 431: 931-945.

Koldobskaya Y, Duguid EM, Shechner DM, Suslov NB, Ye JD, Sidhu SS, Bartel DP, Koide S, Kossiakoff AA, Piccirilli JA. 2011. A portable RNA sequence whose recognition by a synthetic antibody facilitates structural determination. Nat Struct Mol Biol 18: 100-106.

Kugel JF, Goodrich JA. 2012. Non-coding RNAs: key regulators of mammalian transcription. Trends Biochem Sci 37: 144-151.

Kuo PY, Sherwood JK, Saltzman WM. 1998. Topical antibody delivery systems produce sustained levels in mucosal tissue and blood. Nat Biotechnol 16: 163-167.

Laird-Offringa IA, Belasco JG. 1996. In vitro genetic analysis of RNAbinding proteins using phage display libraries. Methods Enzymol 267: $149-168$.

Lee ES, Jeong MS, Singh R, Jung J, Yoon H, Min JK, Kim KH, Hong HJ. 2012. A chimeric antibody to L1 cell adhesion molecule shows therapeutic effect in an intrahepatic cholangiocarcinoma model. Exp Mol Med 44: 293-302.

Lee JY, Park H, Bak G, Kim KS, Lee Y. 2013. Regulation of transcription from two ssrS promoters in 6S RNA biogenesis. Mol Cells 36: 227-234.

Lefranc MP. 2011. IMGT unique numbering for the variable (V), constant (C), and groove (G) domains of IG, TR, MH, IgSF, and MhSF. Cold Spring Harb Protoc 2011: 633-642.

McCormick M. 1987. Sib selection. Methods Enzymol 151: 445-449.

Neve RM, Chin K, Fridlyand J, Yeh J, Baehner FL, Fevr T, Clark L, Bayani N, Coppe JP, Tong F, et al. 2006. A collection of breast cancer cell lines for the study of functionally distinct cancer subtypes. Cancer Cell 10: 515-527.

Pokkuluri PR, Bouthillier F, Li Y, Kuderova A, Lee J, Cygler M. 1994. Preparation, characterization and crystallization of an antibody Fab fragment that recognizes RNA: crystal structures of native Fab and three Fab-mononucleotide complexes. J Mol Biol 243: 283-297.

Ryu CJ, Padlan EA, Jin BR, Yoo OJ, Hong HJ. 1996. A humanized antibody with specificity for hepatitis B surface antigen. Hum Antibodies Hybridomas 7: 113-122.

Schier R, Bye J, Apell G, McCall A, Adams GP, Malmqvist M, Weiner LM, Marks JD. 1996. Isolation of high-affinity monomeric human anti-c-erbB-2 single chain Fv using affinity-driven selection. J Mol Biol 255: 28-43.

Stollar BD. 1980. The experimental induction of antibodies to nucleic acids. Methods Enzymol 70: 70-85.

Tiedge H, Chen W, Brosius J. 1993. Primary structure, neural-specific expression, and dendritic location of human BC200 RNA. $J$ Neurosci 13: 2382-2390.

Ullu E, Tschudi C. 1984. Alu sequences are processed 7SL RNA genes. Nature 312: 171-172.

Walter P, Blobel G. 1982. Signal recognition particle contains a 7S RNA essential for protein translocation across the endoplasmic reticulum. Nature 299: 691-698.

Wan Y, Kertesz M, Spitale RC, Segal E, Chang HY. 2011. Understanding the transcriptome through RNA structure. Nat Rev Genet 12: 641-655.

Wan Y, Qu K, Ouyang Z, Kertesz M, Li J, Tibshirani R, Makino DL, Nutter RC, Segal E, Chang HY. 2012. Genome-wide measurement of RNA folding energies. Mol Cell 48: 169-181.

Wilusz JE, Sunwoo H, Spector DL. 2009. Long noncoding RNAs: functional surprises from the RNA world. Genes Dev 23: 1494-1504.

Ye JD, Tereshko V, Frederiksen JK, Koide A, Fellouse FA, Sidhu SS, Koide S, Kossiakoff AA, Piccirilli JA. 2008. Synthetic antibodies for specific recognition and crystallization of structured RNA. Proc Natl Acad Sci 105: 82-87.

Zhao Y, Huang Y, Gong Z, Wang Y, Man J, Xiao Y. 2012. Automated and fast building of three-dimensional RNA structures. Sci Rep 2: 734.

Zuker M. 2003. Mfold web server for nucleic acid folding and hybridization prediction. Nucleic Acids Res 31: 3406-3415. 

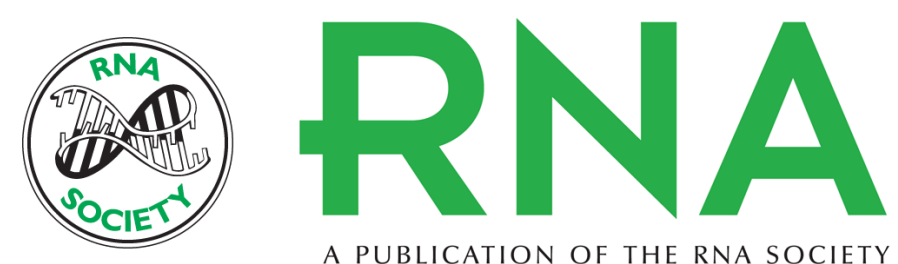

A PUBLICATION OF THE RNA SOCIETY

\section{RNA recognition by a human antibody against brain cytoplasmic 200 RNA}

Euihan Jung, Jungmin Lee, Hyo Jeong Hong, et al.

RNA 2014 20: 805-814 originally published online April 23, 2014

Access the most recent version at doi:10.1261/rna.040899.113

\section{Supplemental http://rnajournal.cshlp.org/content/suppl/2014/04/03/rna.040899.113.DC1 \\ Material}

References This article cites 32 articles, 8 of which can be accessed free at: http://rnajournal.cshlp.org/content/20/6/805.full.html\#ref-list-1

Creative This article is distributed exclusively by the RNA Society for the first 12 months after the Commons License full-issue publication date (see http://rnajournal.cshlp.org/site/misc/terms.xhtml). After 12 months, it is available under a Creative Commons License (Attribution-NonCommercial 4.0 International), as described at http://creativecommons.org/licenses/by-nc/4.0/. top right corner of the article or click here. 